\title{
Communicable Diseases Report, NSW, November and December 2009
}

\section{Communicable Diseases Branch NSW Department of Health}

For updated information, including data and facts on specific diseases, visit www.health.nsw.gov.au and click on Public Health and then Infectious Diseases. The communicable diseases site is available at: http://www.health.nsw.gov.au/publichealth/ infectious/index.asp.

Figure 1 and Tables 1 and 2 show reports of communicable diseases received through to the end of November and December 2009 in New South Wales (NSW).

\section{Measles}

In late October and early November, three cases of measles in children who had returned from a school trip to Vietnam were investigated by the Public Health Unit of Sydney South West Area Health Service. Contacts exposed in a general practitioner's surgery were offered normal human immunoglobulin, and all school and travel contacts were provided with information on the symptoms of measles. Three subsequent cases were identified in local contacts of these cases.

In late November, a woman in her twenties returned from Thailand with measles. The case was considered infectious during the flight and susceptible contacts were provided with information to prevent further spread. No secondary cases were identified.

There were 19 cases of measles reported in NSW residents in 2009, compared with 39 cases in 2008. Most cases of measles in Australia are seen in susceptible travellers and their contacts. People who were born in or since 1966 who have not received two doses of a measles vaccine are likely to be susceptible to measles. Measles vaccine is routinely given to infants at 12 months and at 4 years, and this confers long-lasting immunity.

\section{Hepatitis A}

Public Health Units from South East Sydney Illawarra and Sydney South West Area Health Services investigated two cases of hepatitis A infection in November. Neither person had travelled overseas during the incubation periods, however both reported eating semidried tomatoes in the weeks before becoming ill with hepatitis A. The people may have been linked to a large outbreak of hepatitis A associated with consumption of semidried tomatoes in Victoria (see: http://hnb.dhs.vic.gov.au/web/pubaff/medrel. nsf/LinkView/D8172AF758EDF26ECA25764A002574 DE?OpenDocument).

Hepatitis A virus infection causes inflammation of the liver (hepatitis). Symptoms include feeling unwell, aches and pains, fever, nausea, lack of appetite, abdominal discomfort, followed by dark urine, pale stools and jaundice (yellowing of the eyeballs and skin). Illness usually lasts one to three weeks (although some symptoms can last longer) and is almost always followed by complete recovery. Hepatitis A infection can be transmitted through contaminated food or water, by handling nappies, linen and towels contaminated with the faeces of an infectious person or after direct contact (including sexual contact) with a person in the infectious stage of the illness.

\section{Meningococcal disease}

Twelve cases of meningococcal disease were reported in November and December; three of these (due to serogroup B meningococcus infection) were associated with a year 12 formal in Sydney. The Public Health Unit of South Eastern Sydney Illawarra Area Health Service investigated this cluster and, after consultation with an expert panel, established a special clinic to provide clearance antibiotics to other year 12 students. No further cases emerged.

In previous years, NSW has experienced an increase in cases around the Christmas and New Year party season, presumably because of the increase in close mixing of young people. For example, in 200517 cases were reported in December compared with five cases in November.

There were 91 cases of meningococcal disease reported in NSW in 2009, including four deaths. In 2008, there were 80 cases reported and three deaths. 
A free vaccine is available for infants at 12 months of age. Consequently, serogroup $\mathrm{C}$ meningococcal disease is now mainly seen in adults and in unimmunised children. In NSW this year to date $78 \%$ of cases of meningococcal disease (where serogroup was known) were caused by serogroup B, for which there is no vaccine.

\section{Pandemic (H1N1) 2009 influenza}

Influenza activity in NSW is measured by the number of people who present to emergency departments with influenza-like illness, and the number of patients whose swabs test positive for H1N1 at diagnostic laboratories.

During November and December, influenza activity in NSW declined to just above baseline levels. Most cases of influenza occurring in NSW since May 2009 were due to pandemic (H1N1) influenza 2009 (the virus formerly known as swine flu).

For 2009:

- Presentations to emergency departments peaked mid July with an average of 1300 presentations per week

- 5385 cases with laboratory-confirmed pandemic (H1N1) 2009 influenza were reported in NSW. As most people with influenza do not seek or require testing, this number represents only a small proportion of all true cases of infection in NSW

- 1430 patients with laboratory-confirmed pandemic (H1N1) 2009 influenza were reported to have been admitted to NSW hospitals

- 54 deaths were reported in association with confirmed pandemic (H1N1) 2009 influenza in NSW.

For more information and resources, please visit NSW Health's pandemic influenza (H1N1) 2009 web page: http://www.emergency.health.nsw.gov.au/swineflu/index.asp.

\section{Brucellosis}

A case of brucellosis was reported in November from a person who reported hunting feral pigs in northern NSW. Brucellosis is a systemic bacterial disease transmitted by direct contact with tissues, blood, urine, vaginal discharges, aborted animal foetuses, and by ingestion of raw milk and dairy products from infected animals.

Notifications of human brucellosis are rare as the infection has been eradicated from NSW stock animals. However cases are occasionally reported in people who are exposed overseas, or in pig hunters in the north of the state. There have been four cases reported in NSW in 2009 and one case reported in 2008.

\section{Healthy overseas travel: checklist for travellers}

Some infectious diseases and hazards are more common overseas, especially in less industrialised and tropical countries. A checklist for travellers to developing countries and tropical areas of the world include:

\section{Before you travel:}

- Find out what diseases are prevalent in your destination

Check reputable web links (e.g., the US Centers for Disease Control and Prevention: http://wwwnc.cdc. gov/travel) and talk to your doctor.

\section{- Update your vaccinations}

Regardless of where you go, you might mix with travellers who carry measles or influenza. Pandemic influenza vaccine is now available for everyone over 6 months of age, so consider having the vaccine if you are about to travel. If you were born during or since 1966, make sure that you have had two shots of measles, mumps and rubella (MMR) vaccine. Measles, hepatitis A, typhoid and other infectious diseases are more common in some countries. If you are planning to travel in a tropical or developing country, you may also need a hepatitis A, typhoid, yellow fever or other vaccinations.

\section{- Pack your medicines}

Plan ahead to make sure you have your medications with you. Carry a script or doctor's letter for prescription medicines to avoid any confusion at Customs.

\section{While travelling:}

\section{- Avoid mosquitoborne infections}

Malaria, dengue fever and other mosquito-borne infections can be carried by mosquitoes in tropical areas. Ask your doctor whether you need to take tablets to protect against malaria. Unfortunately, tablets won't stop other mosquito-borne diseases like dengue, so in affected areas, wear light coloured, long sleeves and pants, use insect repellent, and you may need a mosquito net for sleeping.

\section{- Avoid food and waterborne diseases}

In many developing countries infections carried by contaminated food or water are very common. Avoid drinking tap water and ice made from it. Only eat food that is piping hot or fruit that you have peeled yourself.

\section{- Don't touch animals}

Rabies is common in many parts of the world and is carried by biting mammals, such as dogs, cats and bats. Rabies has recently appeared in new places such as Bali. Do not handle stray and wild animals, and avoid bites from any animals. If you are bitten or scratched by an animal, immediately wash the wound thoroughly with soap and water and seek medical attention.

\section{- Follow safety rules}

Injuries are one of the most common hazards for travellers. The safety rules in Australia also make sense when you are overseas. Wear a helmet when on a motor bike or a bicycle, use seat belts in cars, and take extra care when crossing roads. 
Figure 1. Reports of selected communicable diseases, NSW, January 2004 to December 2009, by month of onset.

Preliminary data: case counts in recent months may increase because of reporting delays.

Laboratory-confirmed cases only, except for measles, meningococcal disease and pertussis.

BFV, Barmah Forest virus infection; RRV, Ross River virus infection; lab conf, laboratory confirmed;

Men $\mathrm{Gp} C$ and $\mathrm{Gp} B$, meningococcal disease due to serogroup $C$ and serogroup B infection;

other/unk, other or unknown serogroups.

NB: Multiple series in graphs are stacked, except gastroenteritis outbreaks.

NB: Outbreaks are more likely to be reported by nursing homes and hospitals than by other institutions.

\begin{tabular}{|lr|}
\hline \multicolumn{2}{|c|}{ NSW Population } \\
Male & $50 \%$ \\
$<5$ y & $7 \%$ \\
$5-24$ y & $27 \%$ \\
$25-64$ y & $53 \%$ \\
$65+$ y & $13 \%$ \\
Rural & $46 \%$ \\
\hline
\end{tabular}

Arbovirus infections

aBFV $\square R R V$

\begin{tabular}{|c|c|}
\hline \multicolumn{2}{|c|}{ Oct. 09-Dec. 09} \\
\hline Male & $48 \%$ \\
\hline$<5 y$ & $0.0 \%$ \\
\hline $5-24 y$ & $13 \%$ \\
\hline $25-64$ y & $71 \%$ \\
\hline $65+y$ & $16 \%$ \\
\hline Rural & $92 \%$ \\
\hline
\end{tabular}

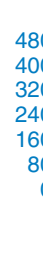

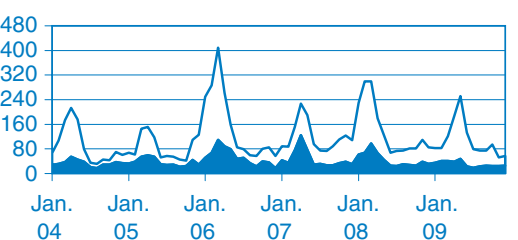

Cryptosporidiosis

\begin{tabular}{lr}
\multicolumn{2}{c}{ Oct. 09-Dec. 09} \\
Male & $51 \%$ \\
$<5$ y & $49 \%$ \\
$5-24$ y & $25 \%$ \\
$25-64$ y & $26 \%$ \\
$65+y$ & $0 \%$ \\
Rural & $65 \%$
\end{tabular}

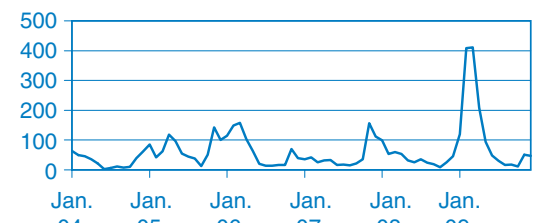

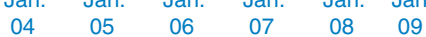

Gastroenteritis outbreaks in institutions

\begin{tabular}{|c|c|}
\hline \multicolumn{2}{|l|}{ Oct. 09-Dec. 09} \\
\hline All outbreaks & 73 \\
\hline Nursing homes 9 & 99 \\
\hline Hospitals & 39 \\
\hline Child care & 28 \\
\hline Schools & 0 \\
\hline Other & 7 \\
\hline
\end{tabular}

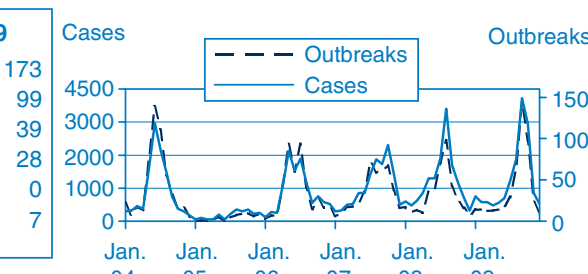

\begin{tabular}{|lr}
\hline Oct. 09-Dec. 09 \\
Male & $80 \%$ \\
$<5$ y & $50 \%$ \\
$5-24$ y & $40 \%$ \\
$25-64$ y & $10 \%$ \\
$65+y$ & $0 \%$ \\
Rural & $40 \%$ \\
\hline
\end{tabular}

Legionnaires' disease

\begin{tabular}{|lr|}
\hline Oct. 09-Dec. 09 \\
Male & $69 \%$ \\
$<5$ y & $0 \%$ \\
$5-24$ y & $0 \%$ \\
$25-64$ y & $41 \%$ \\
$65+y$ & $59 \%$ \\
Rural & $41 \%$ \\
\hline
\end{tabular}

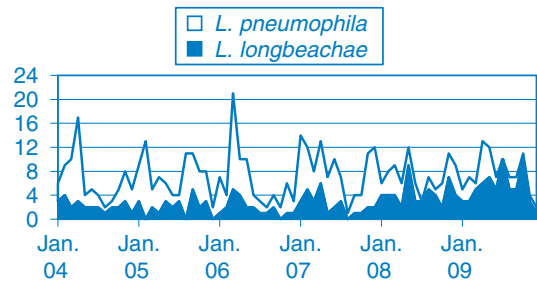

Measles

\begin{tabular}{|lr|}
\hline \multicolumn{2}{|c|}{ Oct. 09-Dec. 09 } \\
Male & $57 \%$ \\
$<5$ y & $0 \%$ \\
$5-24$ y & $71 \%$ \\
$25-64$ y & $29 \%$ \\
$65+y$ & $0 \%$ \\
Rural & $0 \%$ \\
\hline
\end{tabular}

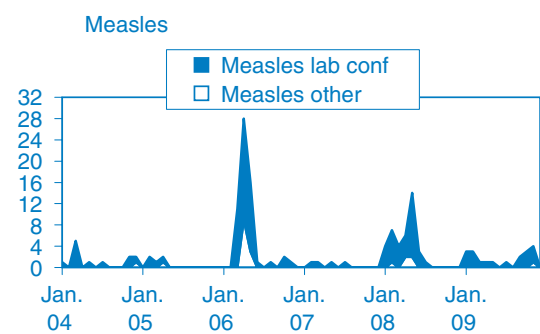

$\begin{array}{llllll}04 & 05 & 06 & 07 & 08 & 09\end{array}$

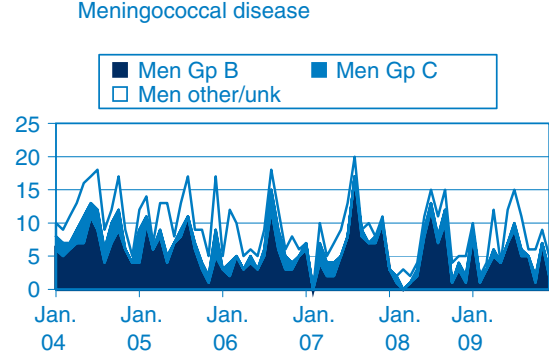

\begin{tabular}{|cr}
\hline \multicolumn{2}{|c}{} \\
\multicolumn{2}{|c|}{ Oct. 09-Dec. 09 } \\
Male & $83 \%$ \\
$<5 y$ & $0 \%$ \\
$5-24$ y & $27 \%$ \\
$25-64$ y & $72 \%$ \\
$65+y$ & $1 \%$ \\
Rural & $14 \%$ \\
\end{tabular}

\section{Gonorrhoea}

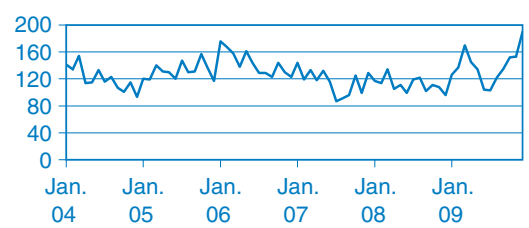

Hepatitis A

Oct. 09-Dec. 09

$\begin{array}{lr}\text { Male } & 44 \% \\ <5 y & 8 \% \\ 5-24 \text { y } & 48 \% \\ 25-64 \text { y } & 36 \% \\ 65+y & 8 \% \\ \text { Rural } & 24 \%\end{array}$

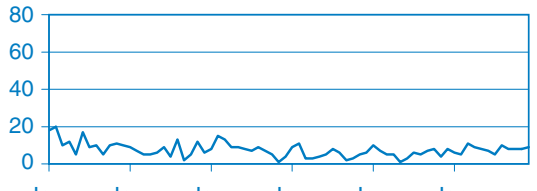

Jan. Jan. Jan. Jan. Jan. Jan. Month of onset
Pertussis

\begin{tabular}{lr}
\hline Oct. 09-Dec. 09 \\
Male & $44 \%$ \\
$<5$ y & $16 \%$ \\
$5-24$ y & $49 \%$ \\
$25-64$ y & $29 \%$ \\
$65+y$ & $6 \%$ \\
Rural & $55 \%$
\end{tabular}

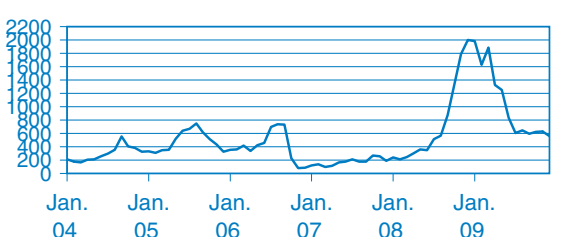




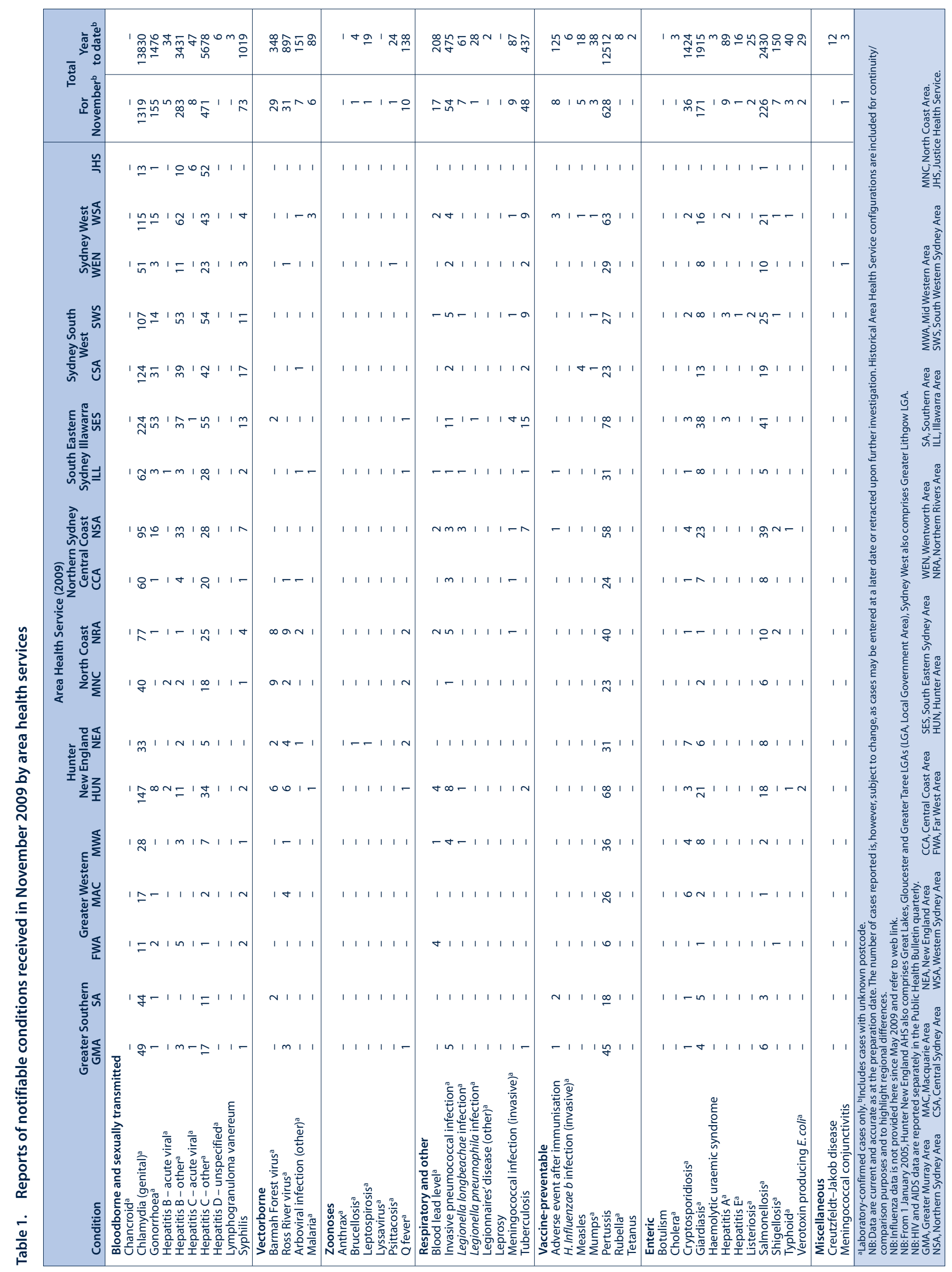




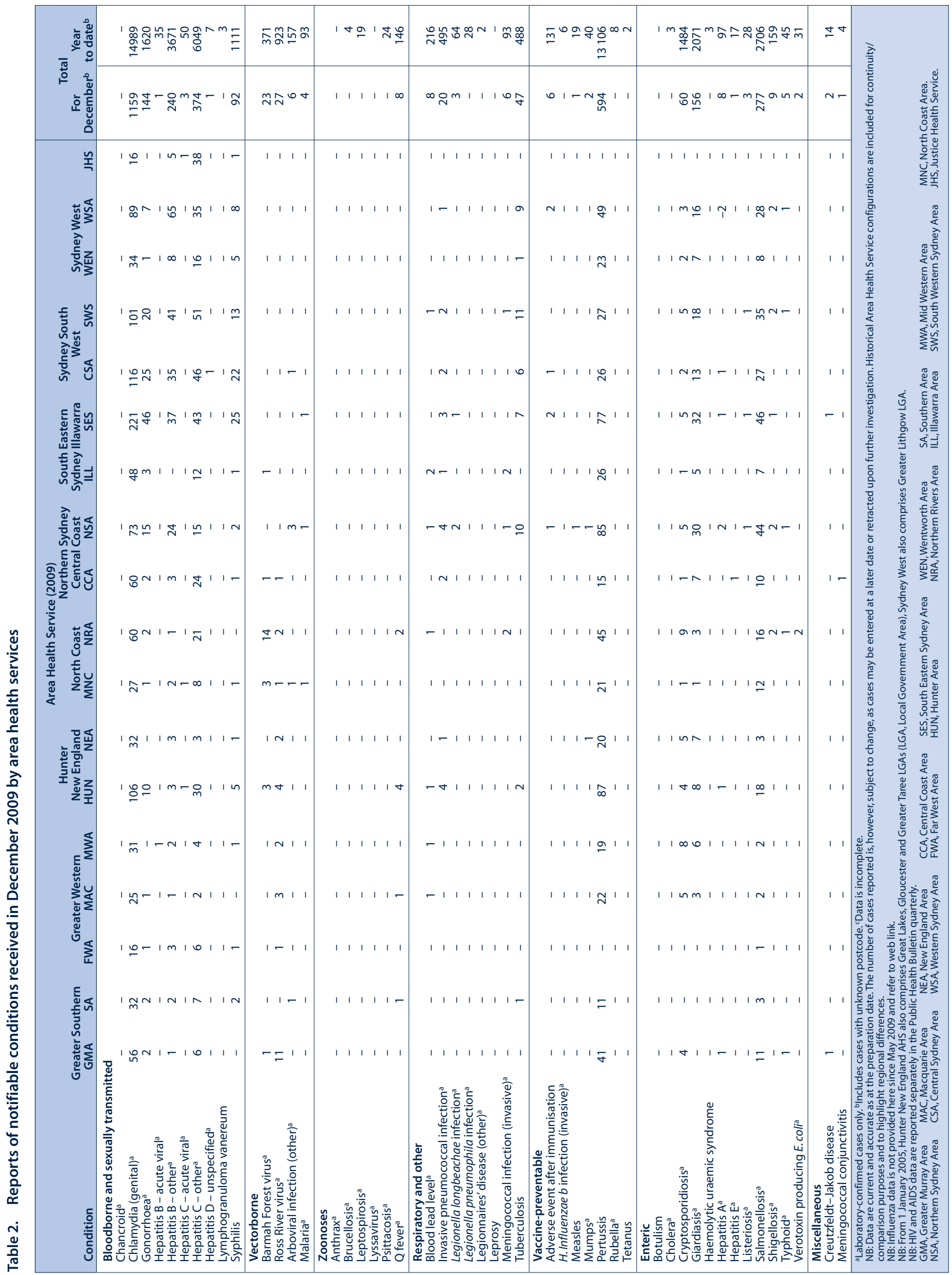

